\title{
Propagation of Bessel Beams along Atomic Columns in Crystal: a Bloch Wave and Multi-slice Analysis
}

Vincenzo Grillo $^{1,2}$, Enzo Rotunno², Benjamin McMorran ${ }^{3}$, Stefano Frabboni1 ${ }^{1,4}$

1. CNR-Istituto Nanoscienze, Centro S3, Via G. Campi 213/a, I-41125 Modena, Italy

2. CNR-IMEM Parco Area delle Scienze 37/A, I-43124 Parma, Italy

3. Department of Physics, University of Oregon, Eugene, Oregon, USA

4. Dipartimento FIM, Università di Modena e Reggio Emilia, Via G. Campi 213/a, I-41125 Modena, Italy

After the recent introduction of holographic methods to produce Bessel beams, it is becoming interesting to understand the theoretical and applicative potentialities of these beams [1]. One of the most interesting properties of the Bessel beams can be tight back to the fact that they are the Fourier transform of a very small annulus in the Fourier space and are therefore similar to hollow cone illumination [2]. However while hollow cone generation is restricted to $0^{\text {th }}$ order Bessel beams, the holographic approach permits to induce a spiraling phase, as in the most common Vortex beam generated by pitch fork holograms.

We report on a detailed analysis on the propagation of Bessel beams of different orders on a $\mathrm{Ga}$ atomic column in a [100] oriented GaN crystal. The beam average convergence was 15 mrad and the beam energy was $300 \mathrm{KeV}$.

The analyses are based on the comparison between Bloch wave and multislice simulations. Considering the simplicity of the Bessel beam momentum spectrum and the symmetry of the material it is possible to give a description of the propagation as a function of a few states.

This analysis permits a deeper understanding of the channeling phenomena and of the probe intensity oscillation in the propagation direction.

For example in fig 1a,b,c, we can observe the analysis for the $0^{\text {th }}$ order Bessel beam. The probe at the interaction in fig $1 \mathrm{a}$ is represented before the interaction. In fig $1 \mathrm{~b}$ the intensity of the central part of the probe are plotted against the depth. Fig 1c is an effective excitation distribution as a function of the Bloch eigenvalue (all excitations for the same energy/eigenvalue are summed coherently).

The probe intensity oscillation in fig $1 \mathrm{~b}$ is more prominent than what observed in common aperture limited probes. The persistence of oscillation can be explained by observing in fig 1c that the oscillation are the beating between a small number of states (typical of Bessel beam narrow lateral spectrum) and the $1 \mathrm{~s}$ state.

Fig 2 a,b,c and fig 3a,b,c are similar sequence of probe shape, intensity evolution with depth and Bloch spectrum for beams with topological charge $q=1,2$. In this case the symmetry selection rules forbid the excitation of the $1 \mathrm{~s}$. In the case of $q=1$ the resulting spectrum shows many peaks and therefore practically no oscillation. In the case of $q=2$ the selection rules and the limited momentum spectrum allow for only few excited states. As a consequence oscillations are again very clear. Bessel beam can be therefore a useful way to engineer the depth profile of the probe for possible depth resolved experiments. 
References:

[1] V. Grillo, E. Karimi, G C Gazzadi, S Frabboni,M R. Dennis, and R W. Boyd Phys. Rev. X 4, 011013 (2014)

[2] T. Kawasaki, T. Matsutani, T. Ikuta, M. Ichihashi, T. Tanji Ultramicroscopy 110 (2010) 1332-1337

[3] E. Rotunno, M. Albrecht, T. Markurt, T. Remmele and V.Grillo 146 (2014) 62
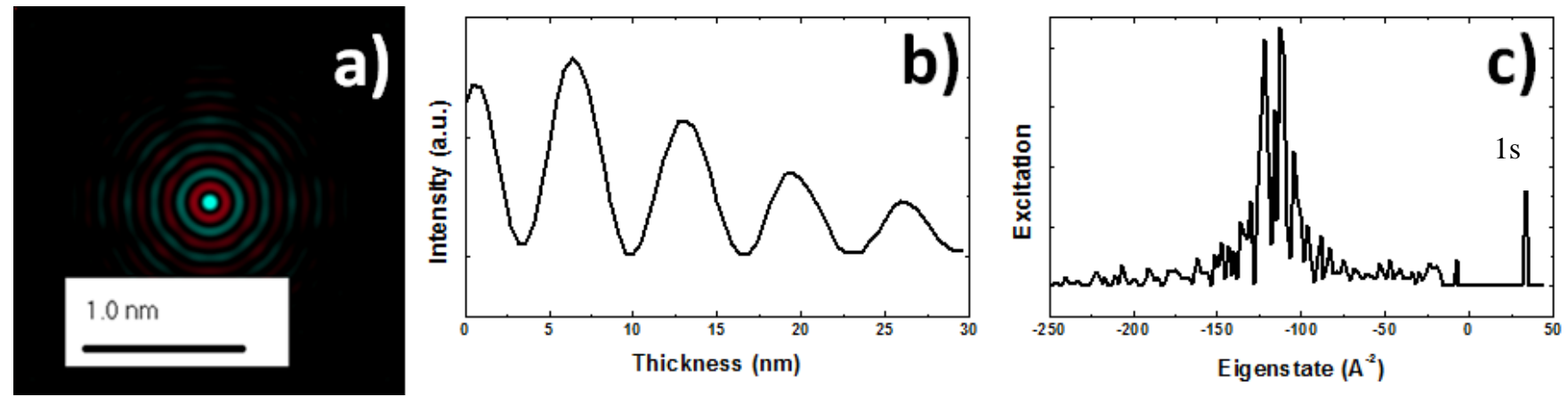

Figure 1. a) Image of the Bessel probe $L=0$ ( hue encodes the phase ). b) Multislice simulation of the probe intensity on the atomic column c) Excitation of the Bloch states vs eigenvalue
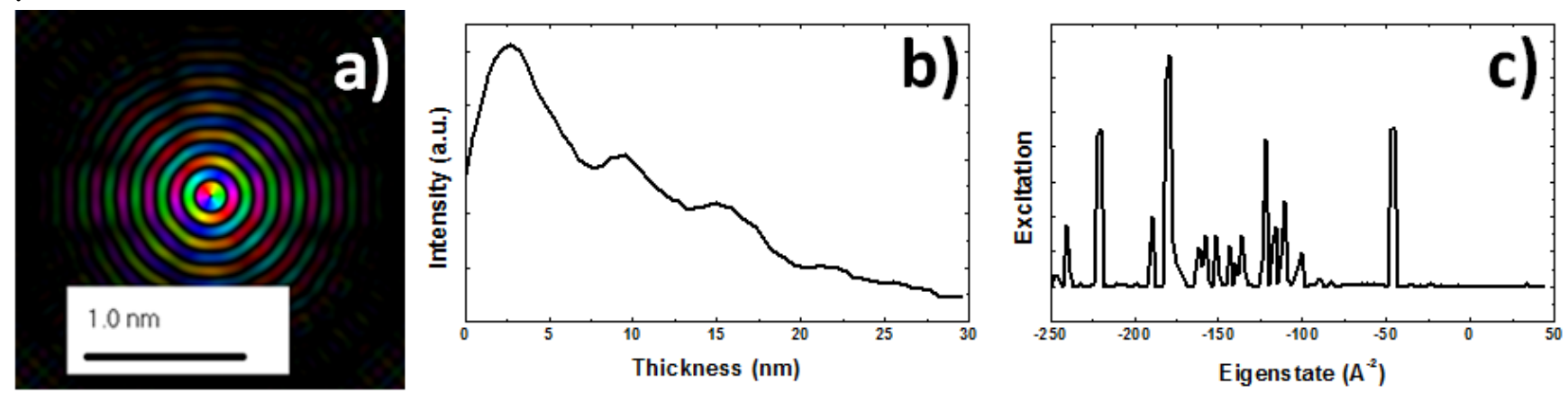

Figure 2. a) Image of the Bessel probe $L=1$ ( hue encodes the phase ). b) Multislice simulation of the probe intensity on the atomic column c) Excitation of the Bloch states vs eigenvalue
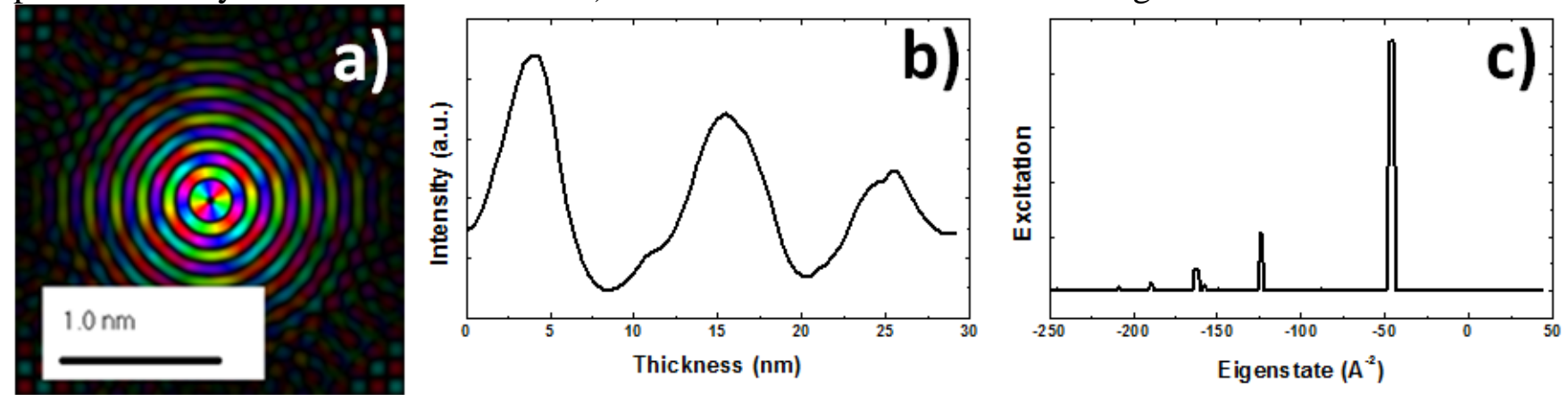

Figure 3 a) Image of the Bessel probe $L=2$ ( hue encodes the phase ). b) Multislice simulation of the probe intensity on the atomic column c) Excitation of the Bloch states vs eigenvalue 